\title{
Quality of Life in Disease-Free Gastric Adenocarcinoma Survivors: Impacts of Clinical Stages and Reconstructive Surgical Procedures
}

\author{
Chi-Cheng Huang $^{a, b}$ Heng-Hui Lien ${ }^{b, c}$ Pa-Chun Wang ${ }^{\text {b, } d ~ J i a o-C h i a o ~ Y a n g ~}{ }^{d}$ \\ Chan-Yeh Cheng $^{a} \quad$ Ching-Shui Huang $^{c}$

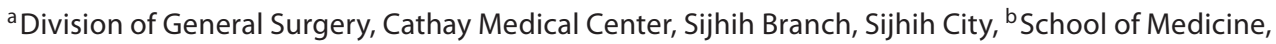 \\ Fu Jen Catholic University, Sinjhuang City, ${ }^{\mathrm{C}}$ Division of General Surgery, Department of Surgery, \\ Cathay Medical Center, Taipei, and d Cathay Medical Research Institute, Taipei, Taiwan
}

\section{Key Words}

Gastric adenocarcinoma - Quality of life, gastric adenocarcinoma suvivors $\cdot$ Reconstructive surgical procedures

\begin{abstract}
Aim: To investigate health-related quality of life data of disease-free gastric adenocarcinoma survivors, with special emphasis on the roles of clinical stages and reconstructive surgical procedures. Methods: We performed a cross-sectional study in 51 disease-free gastric adenocarcinoma patients. The patients had been followed for at least 6 (median 17, range from 6 months to 2 years) months after initial radical surgery. The Taiwan Chinese version of the European Organization for Research and Treatment of Cancer Quality of Life Questionnaire Cancer 30 (EORTC QLQ-C30) and the supplementary gastric cancer module QLQ-STO22 were used as outcomemeasures. Results: Patients with earlier-/advancedstage diseases (American Joint Committee on Cancer stages I and II vs. III and IV) had a similar quality of life in terms of global health status and functional and symptomatic wellbeing. Subtotal gastrectomy outweighed total gastrectomy with better role function and less nausea/vomiting and appetite loss. Multivariate regression analyses also proved that proximal gastric preservation was predictive of better role
\end{abstract}

function, less nausea/vomiting, and less appetite loss. Conclusions: Gastric adenocarcinoma survivors may enjoy a similar life quality, regardless of their original disease stages. Functional preservation may have marginal advantages to improve patients' quality of life by reducing symptomatic nausea, vomiting, and appetite loss postoperatively.

Copyright $\odot 2007$ S. Karger AG, Basel

\section{Introduction}

The health-related quality of life (QoL) has becoming an important component of outcomes in cancer therapy research. A self-reported QoL can be used as a quantitative and subjective approach to assess a multidimensional, yet not easily defined life quality status. The QoL is also an established surrogate end point for the evaluation of cancer therapy [1-3]. A generic questionnaire and a supplementary malignancy-site-specific questionnaire are often used together to enhance survey sensitivity and specificity $[4,5]$.

It is well known that patients who have survived malignant stomach neoplasm surgery may continue to suffer from various symptomatic nutritional or functional problems. QoL outcomes after surgical treatment of stomach neoplasm have drawn much attention these

\section{KARGER}

Fax +4161306 1234 E-Mail karger@karger.ch www.karger.com (c) 2007 S. Karger AG, Basel

0253-4886/07/0241-0059\$23.50/0

Accessible online at:

www.karger.com/dsu
Ching-Shui Huang, MD

Division of General Surgery, Cathay Medical Center

280, Section 4, Jen-Ai Road

106 Taipei (Taiwan)

Tel. +8862 2708 2121, Fax +88622709 2063, E-Mail chishenh@pchome.com.tw 
years. Unfortunately, there is only limited quantitative evidence published in the English literature; QoL outcomes in disease-free gastric adenocarcinoma survivors have not been released in the Asian context.

QoL evaluation in patients with malignant stomach neoplasm should be an integral part in clinical care, especially when the opinions appear to differ with regard to the roles of clinical stages, preservation of stomach, or reconstructions of alimentary continuity in the prediction of postoperative QoL [6-15]. This study used the Taiwan Chinese version of the European Organization for Research and Treatment of Cancer Quality of Life Questionnaire Cancer 30 (EORTC QLQ-C30) and the stomach-specific module QLQ-STO22 to evaluate patients treated for gastric adenocarcinoma [3-5]. The objective of this study was to determine whether clinical stages, reconstructive surgical procedures, and preservation of the stomach would affect the QoL for disease-free patients who had survived surgical treatment.

\section{Patients and Methods}

\section{Study Population}

The study was conducted with a prospective design in a tertiary referral center (Cathay Medical Center, Taipei, Taiwan). Patient information was retrieved from the institute's gastric malignancy registry (years 2002-2003); clinical data were retrospectively reviewed, and the patients were interviewed by means of a self-administered QoL questionnaire. Patients who had pathological, clinical, or radiological evidence of residual or recurrent malignancy were excluded. Patients who had been followed for less than 6 months and those who were under active chemo- and/or radiotherapy were also excluded. The median follow-up time was 17 (range from 6 months to 2 years) months. The study protocol was approved by the Institutional Review Board, and consents of participation from the patients were obtained in advance.

\section{Surgery}

All patients underwent R0 tumor resection and Japanese style D2 lymphadenectomy to eradicate disease at the time of surgery. The choice of total or subtotal gastrectomy depended on site and extent of the lesion, and typically a 5 - to $7-\mathrm{cm}$ safety margin was pursued. The continuity of the digestive tract was reconstructed with either Billroth II gastrojejunostomy for subtotal gastrectomy or Roux-en-Y esophagojejunostomy following total gastrectomy with or without an artificial pouch. D2 lymphadenectomy was performed to include removal of left gastric, hepatic, splenic, and celiac nodes.

\section{Outcome Measurement}

The EORTC QLQ-C30 and the stomach-specific STO22 module (both Taiwan Chinese version) were used as outcome measures; authorization to use was obtained from the EORTC. Prior tests on the instrument reliability, validity, and sensitivity showed that the survey statistical characteristics are equivalent to the original English versions. The EORTC QLQ-C30 comprises a global health status, five multi-item functional scales, and several single-/multi-item symptom scales $[5,16,17]$. Linear transformed QLQ-C30 scores range from 0 to 100, with 100 representing the best global health status. Items evaluating the functional status emerge into physical, role, cognitive, emotional, and social scales separately; higher scores indicate a better QoL. Symptomatic QLQ-C30 scales assess gastric-cancer-related complications such as fatigue, pain, nausea/vomiting, dyspnea, loss of appetite, insomnia, constipation, diarrhea, and perceived financial impact; higher scores indicate worse symptoms. The QLQ-STO22 gastric cancer module replenishes the QLQ-C30 core questionnaire with the aim to enhance survey sensitivity and specificity. All items are symptom related; survey items are categorized into five multiitem scales (dysphagia, eating restriction, pain, reflux, and anxiety) and four single-item scales (dry mouth, body image, hair loss, and taste problems), with higher scores indicating worse symptomatic problems. Scoring, missing data management, and linear transformation were conducted in accordance with EORTC requirements [18].

\section{Administration of Questionnaires}

Eligible patients were enrolled in a cross-sectional manner during their regular outpatient follow-up visit. The patients were well informed of the objectives and confidentiality policy of this study. The questionnaires were self-administered by the patients; no additional aid was given, unless patients met problems in answering specific question items.

\section{Statistics}

The EORTC scores are presented as mean \pm SD. The Wilcoxon rank sum test was used for between-group comparisons. Multivariate regression models were applied to investigate the QoL predictor $[19,20]$. All tests were two-sided, and $p<0.05$ was considered statistically significant.

\section{Results}

\section{Demographic and Clinical Information}

A total of 51 malignant stomach neoplasm patients with pathology-proven adenocarcinoma were available for interview. There were 25 males $(49.0 \%)$ and 26 females $(51.0 \%)$. The median age was 65 (range $26-83$ ) years. The enrollment period spanned from July 1 to December 31 , 2004.

According to the American Joint Committee on Cancer classification of 2002, 15 patients (29.4\%) had stage I, 12 patients (23.5\%) had stage II, 17 patients (33.3\%) had stage III, and 7 patients (13.7\%) had stage IV disease. Total gastrectomy was performed in 14 patients (27.5\%) and subtotal gastrectomy in the remaining 37 patients $(72.5 \%)$. Gastric substitutes were performed with Hunt-LawrenceRodino jejunal pouch in most of the total gastrectomy patients $(\mathrm{n}=12,85.7 \%)$. Braun jejunojejunostomy (10- 
Table 1. Demography and clinical details

\begin{tabular}{|c|c|c|c|c|}
\hline & $\begin{array}{l}\text { Stage I } \\
(\mathrm{n}=15)\end{array}$ & $\begin{array}{l}\text { Stage II } \\
(\mathrm{n}=12)\end{array}$ & $\begin{array}{l}\text { Stage III } \\
(\mathrm{n}=17)\end{array}$ & $\begin{array}{l}\text { Stage IV } \\
(\mathrm{n}=7)\end{array}$ \\
\hline \multicolumn{5}{|l|}{ Gender } \\
\hline Male & 6 & 7 & 9 & 3 \\
\hline Female & 9 & 5 & 8 & 4 \\
\hline \multicolumn{5}{|l|}{ Age, years } \\
\hline Median & 66 & 50 & 66 & 71 \\
\hline Range & $50-81$ & $44-81$ & $26-83$ & $56-82$ \\
\hline \multicolumn{5}{|l|}{ Body mass index, $\mathrm{kg} / \mathrm{m}^{2}$} \\
\hline Median & 23.8 & 26.4 & 24.0 & 22.0 \\
\hline Range & $18.3-37.4$ & $22.2-34.8$ & $19.2-29.3$ & $18.9-24.7$ \\
\hline \multicolumn{5}{|l|}{ Tumor location } \\
\hline Antrum & 3 & 4 & 9 & 2 \\
\hline Body & 9 & 5 & 4 & 2 \\
\hline Cardia & 0 & 1 & 2 & 1 \\
\hline Diffuse & 3 & 2 & 2 & 2 \\
\hline \multicolumn{5}{|l|}{ Reconstruction } \\
\hline Billroth II & 12 & 6 & 12 & 2 \\
\hline Billroth II + Braun & 1 & 1 & 2 & 1 \\
\hline Total gastrectomy & 0 & 0 & 0 & 2 \\
\hline \multicolumn{5}{|l|}{ Total gastrectomy +} \\
\hline Hunt-Lawrence-Rodino & 2 & 5 & 3 & 2 \\
\hline
\end{tabular}

$15 \mathrm{~cm}$ downstream the gastrojejunostomy) was performed in 5 subtotal gastrectomy cases (13.5\%). In general, gastric substitutes were reconstructed in 17 patients (33.3\%; table 1).

\section{Impact of Clinical Stage}

The median global health status/QoL index score was 66.7 (range 33.3-100.0, SD 19.4, interquartile range 16.7) for earlier-stage cases (stages I and II, $\mathrm{n}=27$ ) and 66.7 (range 0.0-100.0, SD 25.3, interquartile range 33.3) for later-stage cases (stages III and IV, $\mathrm{n}=24$ ); the difference did not reach significance $(\mathrm{p}=0.36)$. Patients with earlier stages tended to have higher or equal functional and lower or equal symptom scores in most of the scales, except for dry mouth, but the differences were not remarkable ( $p>0.05$; fig. 1).

\section{Impact of Functional Preservation}

Comparing the QoL between subtotal gastrectomy and total gastrectomy groups, the proximal gastric preservation group outperformed the total gastrectomy group in role function (median 100.0, range 33.3-100.0, SD 19.9, and interquartile range 16.7 for subtotal gastrectomy and median 75.0, range $0-100.0$, SD 26.7, and interquartile range 33.3 for total gastrectomy; $\mathrm{p}=0.02$ ), nausea/vomiting (median 0, range 0-50.0, SD 13.4, and interquartile range 16.7 for subtotal gastrectomy and median 16.7, range $0.0-100.0, \mathrm{SD} 28.2$, and interquartile range 50.0 for total gastrectomy; $\mathrm{p}<0.01$ ), and appetite loss (median 0 , range $0-100.0$, SD 24.6, and interquartile range 33.3 for subtotal gastrectomy and median 33.3 , range $0-100.0$, SD 27.6, and interquartile range 33.3 for total gastrectomy; $\mathrm{p}=0.05$; fig. 2).

After adjustment for gender and age, the multivariate regression models showed that proximal gastric preservation did have positive impacts to enhance the postoperative role function (partial correlation coefficient 0.32 , $\mathrm{p}<0.05$ ), to reduce nausea/vomiting (partial correlation coefficient $-0.37, \mathrm{p}<0.01$ ), and to prevent appetite loss (partial correlation coefficient $-0.30, \mathrm{p}<0.05$ ). In contrast, artificial pouch reconstruction was not predictive of the postoperative QoL. The regression analysis results were compatible with comparative statistics results (table 2).

\section{Discussion}

The importance of health-related QoL following cancer resection cannot be overemphasized in modern surgical oncology. Aside from the biological and pathological consequences of multimodality therapies, functional sta- 


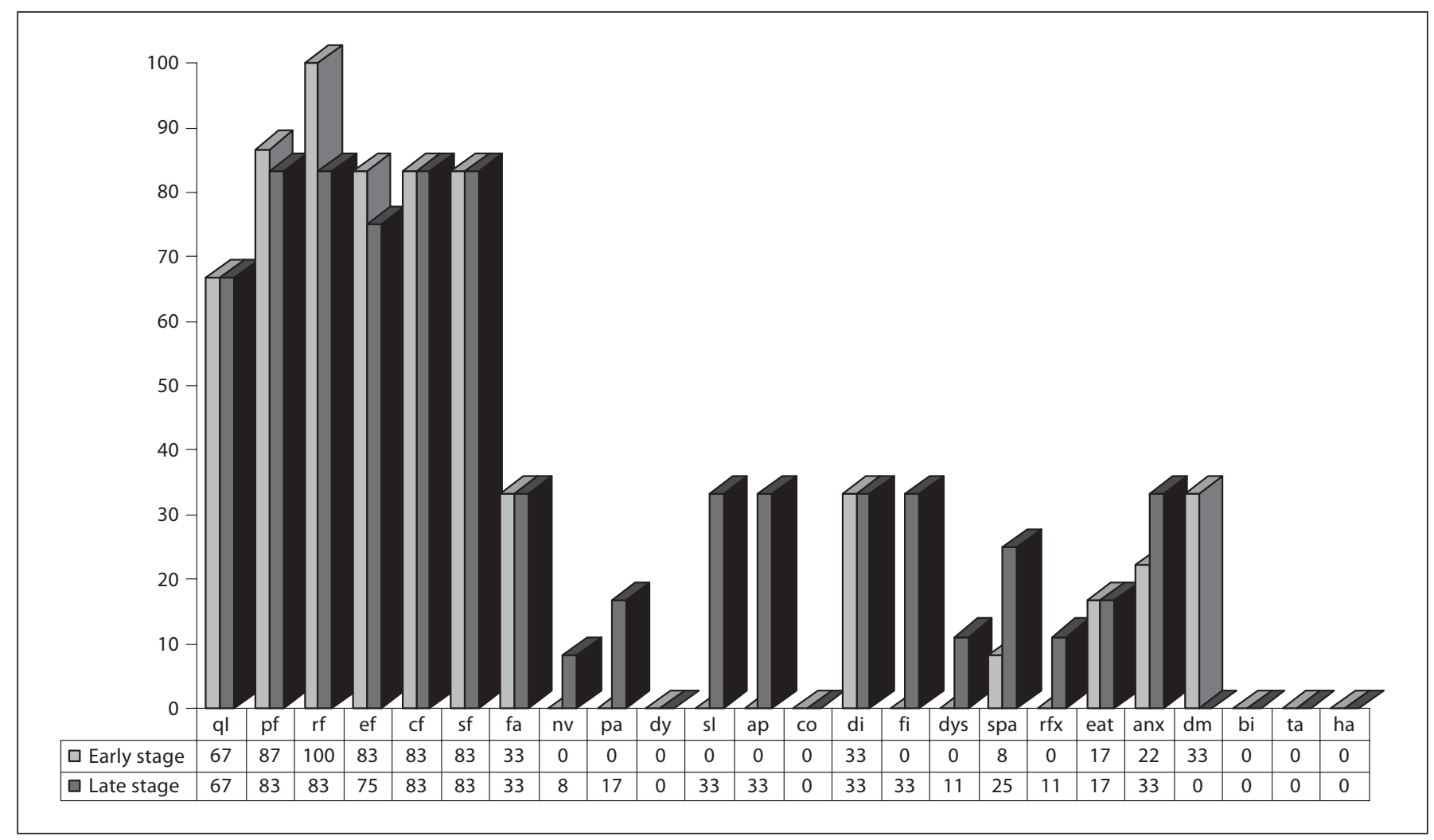

Fig. 1. Earlier versus advanced clinical stages. $\mathrm{ql}=$ Quality of life index; $\mathrm{pf}=$ physical function; $\mathrm{rf}=$ role function; $\mathrm{ef}=$ emotional function; $\mathrm{cf}=$ cognitive function; $\mathrm{sf}=$ social function; fa = fatigue; $\mathrm{nv}=$ nausea/vomiting; pa = pain; $d y=$ dyspnea; $\mathrm{sl}=$ insomnia ap = appetite loss; co = constipation; $\mathrm{di}=$ diarrhea; $\mathrm{fi}=$ perceived financial impact; dys = dysphagia; $\mathrm{spa}=$ stomach-related pain; $\mathrm{rfx}=$ reflux; eat = eating restriction; anx = anxiety; $\mathrm{dm}=$ dry mouth; bi = body image change; ta = taste change; ha = hair loss .

tus and social and psychological aspects of the postoperative QoL may deserve extensive investigations. To obtain quantitative outcome information, in this study we used disease-specific QoL surveys to evaluate diseasefree patients who survived gastric adenocarcinoma surgery. The main interest of this study was to investigate the roles of clinical stage, functional preservation, and reconstructive procedures in predicting the QoL following curative surgery.

Even though comparative analyses between earlier (stages I and II) and later (stages III and IV) clinical stage subgroups did not yield statistic significance in all QoL components, our data showed that patients with early American Joint Committee on Cancer stages (I and II) tended to have a better functional status and less troublesome symptoms than patients with later stages (III and IV). Our data indicated that, except for some symptomatic problems, early- or later-stage patients, once cured, may enjoy similar levels of QoL in general.
There is always a trade-off between disease eradication and functional preservation in oncology surgery. Radical gastric resection is advocated for the prevention of cancer recurrence, while proximal gastric preservation with pouch reconstruction for distal tumors may restore physiological functioning gut transit to reduce postoperative gastrointestinal disturbances. However, opinions regarding surgical options appeared to be diverse in terms of QoL concerns. Davies et al. [11], using five questionnaires to evaluate patient-perceived outcomes 1 year postoperatively, concluded that subtotal gastrectomy could render a better QoL by comparing 26 total gastrectomy patients with 27 subtotal gastrectomy patients (all with radical D2 lymph node dissection) [11]. Jentschura et al. [9] and Gockel et al. [13] found that patients who underwent subtotal gastrectomy displayed better physical functions, while emotional status and social activities did not differ significantly. On the contrary, Diaz De Liano et al. [21] found that the QoL in patients undergoing 


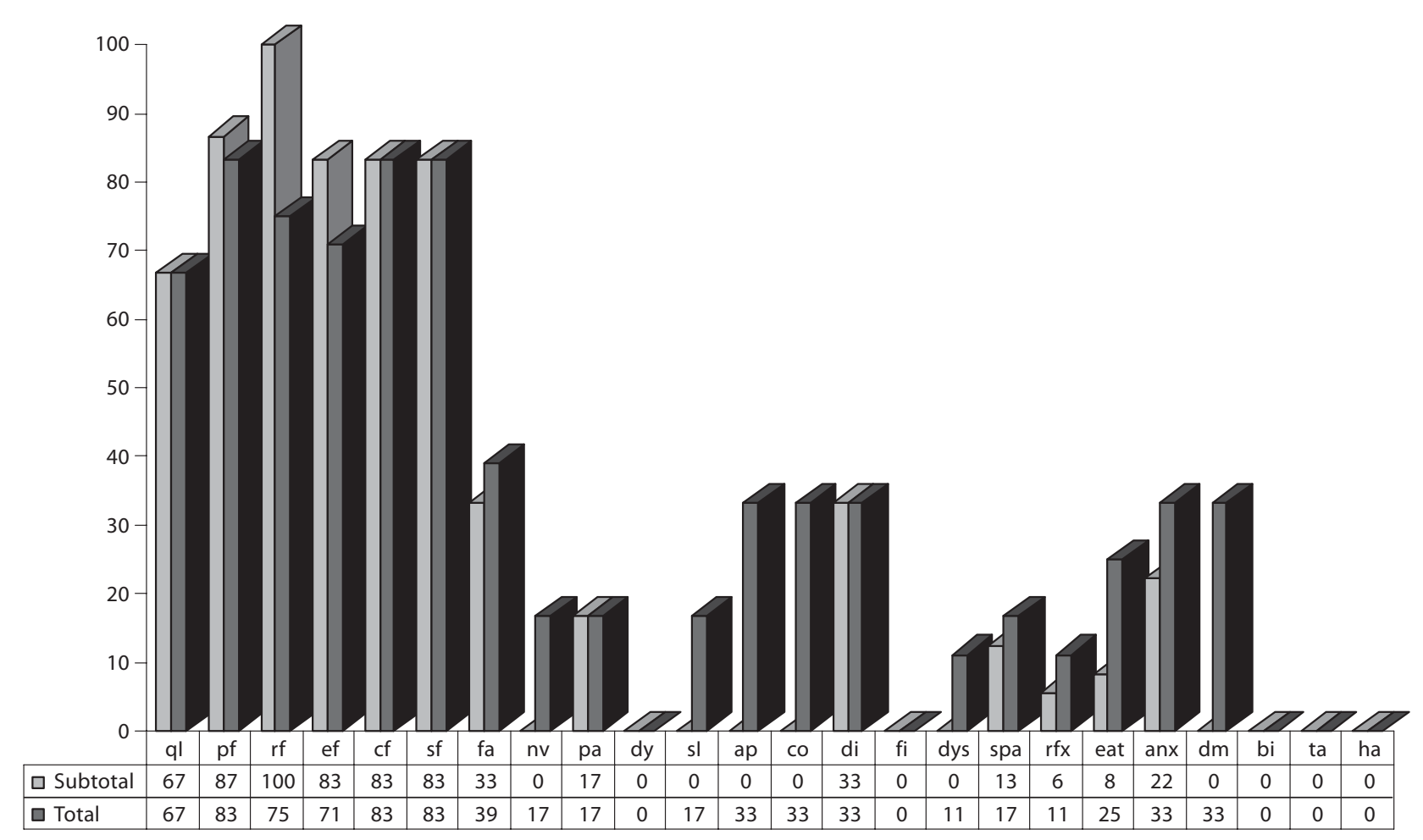

Fig. 2. Subtotal versus total gastrectomy. For explanation of the abbreviations see figure 1 legend.

curative surgery for gastric cancer, regardless of age, was not significantly influenced by type of gastrectomy or whether lymphadenectomy was performed. ThybuschBernhardt et al. [22] found that the global health status was not negatively influenced by D2 lymphadenectomy and extended gastrectomy, rather, patients with splenectomy were evidently more affected. Zieren et al. [10], using an earlier version of the EORTC QLQ-36 to evaluate 71 patients 1 year postoperatively, also did not support the superiority of partial over complete gastrectomy to attain a better QoL. Our data, however, demonstrated a marginal advantage of proximal gastric preservation in rendering better role functioning, less nausea/vomiting, and appetite loss. We agree that an adequate safety margin should still be the primary concern when performing gastric malignancy surgery, but once a safety margin can be secured, functional organ preservation should be considered to provide a better postoperative QoL for our patients.

There have been extensive debates in the literature regarding the role of various pouch designs in restoring the food reservoir function of the stomach, in providing ad-
Table 2. Partial correlation coefficients between QoL scores and predictive variables

\begin{tabular}{llll}
\hline Predictive variables & \multicolumn{2}{l}{ QoL scales } & \\
\cline { 2 - 4 } & $\begin{array}{l}\text { role } \\
\text { function }\end{array}$ & $\begin{array}{l}\text { nausea/ } \\
\text { vomiting }\end{array}$ & $\begin{array}{l}\text { appetite } \\
\text { loss }\end{array}$ \\
\hline Proximal preservation & $0.32^{*}$ & $-0.37^{* *}$ & $-0.30^{*}$ \\
Artificial pouch & 0.13 & 0.01 & 0.19 \\
$\mathrm{R}^{2}$ & 0.13 & 0.22 & 0.1 \\
\hline
\end{tabular}

${ }^{*} \mathrm{p}<0.05 ;{ }^{* *} \mathrm{p}<0.01$.

equate transit, or in reducing reflux/esophagitis symptoms $[6-8,12,14,23-26]$. Tomita et al. [27] investigated patients with Roux-en-Y reconstruction for early gastric cancer (D2 lymph node dissection, curability A). They found that the patients in their IMMC-pIII-positive group had better appetite and ate more with less decrease in body weight than those in the IMMC-pIII-negative group; the patients in the IMMC-pIII-positive group 
clearly had fewer dumping, reflux, esophagitis, nausea/ vomiting, and abdominal pain symptoms. Wu et al. [28] found that patients who underwent Billroth II reconstruction after distal subtotal gastrectomy lost more body weight than those with a Billroth I anastomosis.

Höksch et al. [12], however, found that there was no difference in QoL (using EORTC QLQ-C30 items as outcome measures) for patients with or without pouch reconstruction (41 patients, 1 year follow-up). This contrasts with earlier findings reported by Braga et al. [29] and Jentschura et al. [9] who documented that their subtotal gastrectomy group had a higher dietary energy intake, fewer daily meals, less frequent bowel actions per day, and more body weight gain than the total gastrectomy group. A randomized controlled trial conducted by Svedlund et al. $[8,26]$ confirmed the superiority of subtotal gastrectomy (13 patients) over total gastrectomy (31 patients), but there was no merit from jejunal pouch reconstruction (20 patients) 1 year postoperatively. These authors also concluded that the benefit of pouch reconstruction may eventually emerge when 5-year survival is achieved.

Braun jejunojejunostomy can be used as an extension of the gastric reservoir. This procedure, however, was not routinely performed after subtotal gastrectomy in our institute. In our study group, Braun jejunojejunostomy was indicated only when the afferent loop was extremely redundant to pose a risk of strangulation. We performed a Hunt-Lawrence-Rodino pouch routinely for reconstruction after total gastrectomy; this procedure is technically simple, and its operation time is reasonable. This study did not find obvious QoL advantages in either type of pouch reconstruction. This may probably be attributed to the relatively small sample size involved. To increase the statistical power, more patients would be required in a future study to investigate the possible beneficial effect of a gastric reservoir on subjective outcomes.

QoL investigation is complementary to traditional morbidity/mortality studies in cancer therapy research. Even though the interpretation of data might be limited by subjects' different follow-up intervals, this study provided valuable QoL information for our patients with gastric adenocarcinoma who had survived successful treatment. The outcome information provided by EORTC QLQ-C30 and QLQ-STO22 in this study enhances understandings of the well-being of gastric adenocarcinoma survivors in our institute. We found these individuals may enjoy similar levels of global health, functional status, and symptom-free daily life, regardless of their original disease stage. Our data have shown that proximal gastric preservation may have marginal advantages to improve patients' QoL by improving role function and reducing nausea/vomiting and appetite loss postoperatively. The QoL effect of artificial pouch reconstruction requires further investigation.

\section{Acknowledgments}

This study was supported by Cathay Medical Center research grant CMRI-9302. We thank EORTC for the authorization to use QLQ-C30 and QLQ-STO22. We also thank Prof. Wei-Chu Chie, Institute of Preventive Medicine, National Taiwan University, Taipei, for her technical support in using the Taiwan Chinese version of EORTC questionnaires.

\section{References}

1 Spitzer WO, Dobson AJ, Hall J, Chesterman E, Levi J, Shepherd R, Battista RN, Catchlove BR: Measuring the quality of life of cancer patients: a concise QL-index for use by physicians. J Chronic Dis 1981;34:585-597.

-2 Eypasch E, Williams JI, Wood-Dauphinee S, Ure BM, Schmulling C, Neugebauer E, Troidl H: Gastrointestinal Quality of Life Index: development, validation and application of a new instrument. Br J Surg 1995;82:216-222.

- 3 Aaronson NK, Ahmedzai S, Bergman B, et al: The European Organization for Research and Treatment of Cancer QLQ-C30: a quality-of-life instrument for use in international clinical trials in oncology. J Natl Cancer Inst 1993;85:365-376.
-4 Vickery CW, Blazeby JM, Conroy T, Arraras J, Sezer O, Koller M, Rosemeyer D, Johnson CD, Alderson D; EORTC Quality of Life Group: Development of an EORTC diseasespecific quality of life module for use in patients with gastric cancer. Eur J Cancer 2001; 37:966-971.

-5 Blazeby JM, Conroy T, Bottomley A, Vickery C, Arraras J, Sezer O, Moore J, Koller M, Turhal NS, Stuart R, Van Cutsem E, D’haese S, Coens C; European Organisation for Research and Treatment of Cancer Gastrointestinal and Quality of Life Groups: Clinical and psychometric validation of a questionnaire module, the EORTC QLQ-STO 22, to assess quality of life in patients with gastric cancer. Eur J Cancer 2004;40:2260-2268.
6 Röder JD, Herschbach P, Henrich G, Nagel M, Böttcher K, Siewert JR: The quality of life after total gastrectomy for stomach carcinoma. Esophagojejunal plication with pouch versus esophagojejunostomy without pouch (in German). Dtsch Med Wochenschr 1992; 117:241-247.

$\checkmark 7$ Buhl K, Lehnert T, Schlag P, Herfarth C: Reconstruction after gastrectomy and quality of life. World J Surg 1995;19:558-564.

8 Svedlund J, Sullivan M, Liedman B, Lundell L, Sjödin I: Quality of life after gastrectomy for gastric carcinoma: controlled study of reconstructive procedures. World J Surg 1997; 21:422-433. 
9 Jentschura D, Winkler M, Strohmeier N, Rumstadt B, Hagmüller E: Quality-of-life after curative surgery for gastric cancer: a comparison between total gastrectomy and subtotal gastric resection Hepatogastroenterology 1997;44:1137-1142.

-10 Zieren HU, Zippel K, Zieren J, Müller JM: Quality of life after surgical treatment of gastric carcinoma. Eur J Surg 1998;164:119125.

-11 Davies J, Johnston D, Sue-Ling H, Young S, May J, Griffith J, Miller G, Martin I: Total or subtotal gastrectomy for gastric carcinoma? A study of quality of life. World J Surg 1998; 22:1048-1055.

12 Höksch B, Ablassmaier B, Zieren J, Müller JM: Quality of life after gastrectomy: Longmire's reconstruction alone compared with additional pouch reconstruction. World J Surg 2002;26:335-341.

13 Gockel I, Pietzka S, Junginger T: Quality of life after subtotal resection and gastrectomy for gastric cancer (in German). Chirurg 2005;76:250-257.

14 Lehnert T, Buhl K: Techniques of reconstruction after total gastrectomy for cancer. Br J Surg 2004;91:528-539.

$\checkmark 15$ Kaptein AA, Morita S, Sakamoto J: Quality of life in gastric cancer. World J Gastroenterol 2005;11:3189-3196.

$\checkmark 16$ Chie WC, Chang KJ, Huang JS, Kuo WH: Quality of life of breast cancer patients in Taiwan: validation of the Taiwan Chinese version of the EORTC QLQ-C30 and EORTC BR-23. Psychooncology 2003;12:729-735.
17 Chie WC, Yang $\mathrm{CH}$, Hsu C, Lai CC: Introduction of the EORTC disease-specific quality of life questionnaires for cancer patients. Formos J Med 2002;6:220-227.

18 Fayers PM, Aaronson NK, Bjordal K, Groenvold M, Curran D, Bottomley A: EORTC QLQ-C30 Scoring Manual, ed 3. Brussels, EORTC, 2001, pp 5-13.

19 Hjermstad MJ, Fayers PM, Bjordal K, Kaasa S: Using reference data on quality of life - the importance of adjusting for age and gender, exemplified by the EORTC QLQ-C30 (+3). Eur J Cancer 1998;34:1381-1389.

20 Hjermstad MJ, Fayers PM, Bjordal K, Kaasa S: Health-related quality of life in the general Norwegian population assessed by the European Organization for Research and Treatment of Cancer Core Quality-of-Life Questionnaire: the QLQ-C30 (+ 3). J Clin Oncol 1998;16:1188-1196.

21 Diaz De Liano A, Oteiza Martinez F, Ciga MA, Aizcorbe M, Cobo F, Trujillo R: Impact of surgical procedure for gastric cancer on quality of life. Br J Surg 2003;90:91-94.

22 Thybusch-Bernhardt A, Schmidt C, Küchler T, Schmid A, Henne-Bruns D, Kremer B: Quality of life following radical surgical treatment of gastric carcinoma. World J Surg 2000;24:497.

23 Troidl H, Kusche J, Vestweber KH, Eypasch E, Maul U: Pouch versus esophagojejunostomy after total gastrectomy: a randomized clinical trial. World J Surg 1987;11:699-712.
24 Nakane Y, Okumura S, Akehira K, Okamura S, Boku T, Okusa T, Tanaka K, Hioki K: Jejunal pouch reconstruction after total gastrectomy for cancer. A randomized controlled trial. Ann Surg 1995;222:27-35.

25 Bozzetti F, Bonfanti G, Castellani R, Maffioli L, Rubino A, Diazzi G, Cozzaglio L, Gennari L: Comparing reconstruction with Roux-en-Y to a pouch following total gastrectomy. J Am Coll Surg 1996;183:243248.

26 Svedlund J, Sullivan M, Liedman B, Lundell L: Long-term consequences of gastrectomy for patient's quality of life: the impact of reconstructive techniques. Am J Gastroenterol 1999;94:438-445.

-27 Tomita R, Fujisaki S, Tanjoh K, Fukuzawa M: Relationship between jejunal interdigestive migrating motor complex and quality of life after total gastrectomy with Roux-en-Y reconstruction for early gastric cancer. World J Surg 2003;27:159-163.

28 Wu CW, Hsieh MC, Lo SS, Lui WY, P'eng FK: Quality of life of patients with gastric adenocarcinoma after curative gastrectomy. World J Surg 1997;21:777-782.

29 Braga M, Molinari M, Zuliani W, Foppa L, Gianotti L, Radaelli G, Cristallo M, Di Carlo V: Surgical treatment of gastric adenocarcinoma: impact on survival and quality of life. A prospective ten year study. Hepatogastroenterology 1996;43:187-193. 